Vol.: $1 \quad$ Issue: 1 Date: December 2017 Received: 26.12.2017 Accepted: 29.12.2017 Final Version: 31.12.2017

ISVOS Journal, 2017, 1(1): 51-58

\title{
Improved Compound Multiphase Waveforms with Additional Amplitude Modulation (periodic mode) for Marine Radars
}

\author{
V. Koshevyy a , O. Pashenko a,1 \\ ${ }^{a}$ National University Odessa Maritime Academy, Odessa, Ukraine
}

\begin{abstract}
This paper has presented the basis of a compound multiphase waveform design with additional amplitude modulation, capable of controlling a waveform pick-factor, suitable for use with marine radar. The waveform shows good Doppler tolerance, with the low side-lobes performance maintained over the central zone of an ambiguity function. A clear waveform design procedure has been presented that does not require the implementation of numerical optimization procedures. It has been shown that a compromise between side-lobes suppression and the value of pick-factor can be found. These waveforms allow achieving better results as compared to compound multiphase waveforms without additional amplitude modulation under mismatched weighting filtering. In this article we considered periodic mode of Radar.
\end{abstract}

Keywords: "Composite signal, Ambiguity function, Cross-ambiguity function, Amplitude modulation, Periodic mode"

\section{Introduction}

The compound multiphase signal consists of multiplication of two sequences; each of them is a signal with a quadratic variation of phases. Expressions for complex amplitudes for the base (snB) and external (snV) signal and final multiphase compound signal's sequence (sn) are follows [1,2]:

$$
\begin{gathered}
s_{n}^{B}=\exp \left\{j \frac{\pi}{4} \alpha^{\prime}\left[2\left(n-N_{B} E\left[n / N_{B}\right]+1\right)-\left(N_{B}+\mu_{0 B}\right)\right]^{2}\right\} \\
s_{n}^{V}=\exp \left\{j \frac{\pi}{4} \beta^{\prime}\left[2\left[E\left[n / N_{B_{1}}\right]-N_{V} E\left[\frac{E\left[n / N_{B_{1}}\right]}{N_{V}}\right]+1\right]-\left(N_{V}+\mu_{0 V}\right)\right]^{2}\right\}, n=\overline{0, N-1} \\
s_{n}=s_{n}{ }^{V} \cdot s_{n}{ }^{B}
\end{gathered}
$$

where $\alpha^{\prime}=\alpha T_{0}^{2} ; \beta^{\prime}=\beta\left(T_{0} N_{B}\right)^{2} ; \alpha, \beta, \mu_{0 B}, \mu_{0 \mathrm{v}}, N_{B 1}$ - parameters of phase modulation; $T_{0}$ - duration of one pulse; $N_{B}$ T0- period of the base sequence; $N_{V}$ T0-period of the external sequence; $N T 0=N_{B} N_{V}$ T0-period of the sequence; $E[x]$ - integer part of $x$. If $N_{B}$ is even so $\mu_{0 B}=0$, if $N_{B}$ is no even so $\mu_{0 B}=1 . \mu_{0 V}$ is formed by the same way. As opposite to previous prepared article (we considered aperiodic mode of Radar) [3], in this article we considered periodic mode, which is important for building continuous wave radar. For illustration three types of periodic compound multiphase signals were considered. The length of sequence is $N=324\left(N_{B}=18, N_{V}=18\right), \mu_{0 B}=\mu_{0 V}=0$ with parameters:

1. $\alpha^{\prime}=-1 / N_{B}, \beta^{\prime}=1 / N_{B}^{2}$. The signal with such parameters has a maximum ratio of the free zone (FZ) area around the central peak $(\mathrm{CP})$ to the $\mathrm{CP}$ topographic section area (on the zero level) [2].

2. $\quad \alpha^{\prime}=-1 / N_{B}, \beta^{\prime}=1 / N_{B}$. The side-lobes level is increased in the FZ region for these parameters in comparative to the case 1). But the Autocorrelation Function (ACF) has the lowest side-lobes level for its entire length. It should also be noted that FZ decreases around CP of Ambiguity Function (AF) [4].

\footnotetext{
${ }^{1}$ Corresponding author. Tel.: +0-000-000-0000 ; fax: +0-000-000-0000 .

E-mail address: vmkoshevyy@gmail.com
} 
$\alpha^{\prime}=-1 / N_{B}, \beta^{\prime}=0$.

At the same time the periodic compound multiphase signals with the length of sequence $N=306\left(N_{B}=18, N_{V}=17\right), \alpha^{\prime}=1 / N_{B}, \beta^{\prime}$ $=2 / N_{V}, \mu_{0 B}=0, \mu_{0 V}=1$ were considered.

Signals with an additional amplitude modulation (AM) can be described by the following expression:

$$
\begin{gathered}
s_{n}^{a m}=s_{n} * v_{n} \\
v_{n}=v_{n-E\left[n / N_{B}\right] N_{B}}^{b} \cdot v_{E\left[\frac{n}{N_{V}}\right]+1}^{v}, n=\overline{0 \div N-1,}
\end{gathered}
$$

where $s_{n}{ }^{a m}$ - compound multiphase signal with an additional AM; $s_{n}$ - complex envelope of the signal; $v_{n}^{b}, v_{n}{ }^{v}-$ weighting coefficients for the base and external sequences.

The expression of the AF for the periodic compound multiphase signal with an additional AM has the form:

$$
\chi_{s s}(k, l)=\sum_{n=0}^{N-1} s_{n}^{a m} \cdot s_{(n+k)}^{a m} \cdot e^{i \frac{2 \pi l n}{4 N}}
$$

where $k$ - discrete values of time delay with the selected step $T_{0} ; l$ - discrete values of frequency.

It's convenient to use the pick-factor parameter for describing behaviour of AM modulation [5]. It is defined as the ratio:

$$
\xi=\frac{\left|s_{n_{-} \max }^{\text {am }}\right|^{2} N}{\sum_{n=0}^{N-1}\left|s_{n}^{a m}\right|^{2}}
$$

The law of the changing weighting function sin is considered in the paper. This function allows controlling the behaviour of AF. Its advantage is possibility to control the signal peak-factor and the side-lobes level of AF.

$$
\begin{aligned}
& v_{n}{ }^{B}=\sin \left[\pi\left(\frac{y_{B}}{N_{B}+1}+\frac{n}{N_{B}+z_{B}}\right)\right], n=0 \div N_{B}-1 \\
& v_{n}{ }^{V}=\sin \left[\pi\left(\frac{y_{V}}{N_{V}+1}+\frac{n}{N_{V}+z_{V}}\right)\right], n=0 \div N_{V}-1
\end{aligned}
$$

where $y_{B}, y_{V}$ - the positive numbers for the base and external sequences, respectively, varies in the range $1 \leq y_{B}<\frac{N_{B}+1}{2}$, $1 \leq y_{V}<\frac{N_{V}+1}{2} ; z_{B}, z_{V}$ - variables for the basic and external sequences are $z_{B}=\frac{2 y_{B} N_{B}-\left(N_{B}+1\right)}{N_{B}+1-2 y_{B}}, z_{V}=\frac{2 y_{V} N_{V}-\left(N_{V}+1\right)}{N_{V}+1-2 y_{V}}$.

The formula (8) describes other one of the possible weighting functions for the signals (3):

$$
\begin{aligned}
& v_{n}{ }^{B}=\left(\sin \left[\pi\left(\frac{y_{B}}{N_{B}+1}+\frac{n}{N_{B}+z_{B}}\right)\right]\right)^{2}, n=0 \div N_{B}-1 \\
& v_{n}{ }^{V}=\left(\sin \left[\pi\left(\frac{y_{V}}{N_{V}+1}+\frac{n}{N_{V}+z_{V}}\right)\right]\right)^{2}, n=0 \div N_{V}-1
\end{aligned}
$$

The structure of AF of multiphase compound signals with an additional AM is compared with Cross-ambiguity function (CAF) compound multiphase signals without AM, but with mismatched treatment using weighing functions $\sin ^{(7)}$ and $\sin ^{2}(8)$. On the left upper part of the Figure the body of CAF in the central peak area is represented. Lower the sections of CAF along axe of range for different discrete values of Doppler frequency $(l=0, l=1, l=2, l=3, l=4)$ in FZ (central peak area) are represented. On the right upper part of the Figure the law of the phase modulation of the composite multiphase signal is illustrated. Lower the Cross correlation function (section of CAF by the plane $l=0$ ) on all it's length is presented. And more lower the sections of CAF along Doppler axe b y the planes $(k=0 ; k=1 ; k=2 ; k=3)$. 


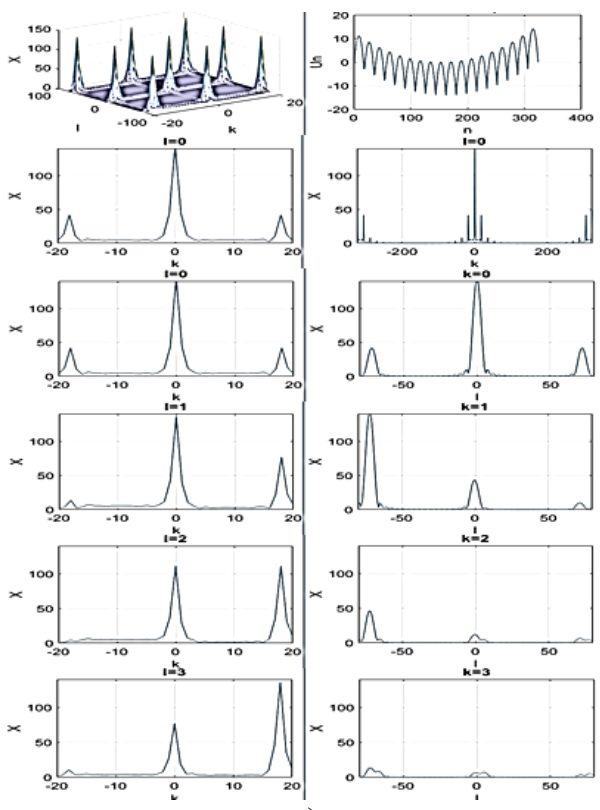

a)
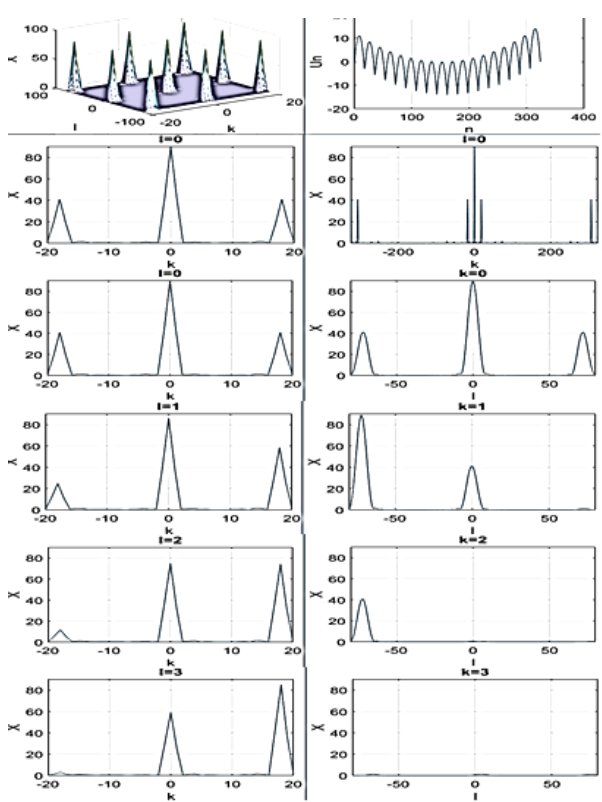

b)

Fig 1. a) The body of the CAF of the periodic multiphase compound signal without an additional AM,

$\mathrm{N}=324(\mathrm{NB}=18, \mathrm{NV}=18) \alpha^{\prime}=-1 / \mathrm{NB}, \beta^{\prime}=1 / \mathrm{NB}, \mu 0 \mathrm{~V}=\mu \mathrm{0B}=0, \mathrm{NB1}=\mathrm{NB}$, with the weighting function $\sin (\mathrm{yB}=\mathrm{yV}=1)$, phases of the signal, sections of the body of the CAF; b) the body of the AF periodic multiphase compound signal with an additional AM, N=324 $(\mathrm{NB}=18, \mathrm{NV}=18) \alpha^{\prime}=-1 / \mathrm{NB}, \beta^{\prime}=1 / \mathrm{NB}, \mu 0 \mathrm{~V}=\mu 0 \mathrm{~B}=0, \mathrm{NB} 1=\mathrm{NB}$, with the weighting coefficients $\sin (\mathrm{yB}=\mathrm{yV}=1)$, phases of the signal, sections of the body of the AF.

As a result (Fig.1,2,3), side-lobes level is significantly reduced after using the periodic compound multiphase signals with an additional AM. CP area isn't expanded significantly. By increasing the $y_{B}$ and $y_{V}$, the value of peak-factor is decreasing. By changing the parameters $y_{B}$ and $y_{V}$ we can control the peak-factor of the signal. Table 1 shows these values:

Table 1. The peak-factor dependence from parameters $y_{B}=y_{V}$ for periodic multiphase compound signal with an additional AM, $N=324\left(N_{B}=18, N_{V}=18\right) \alpha \alpha^{\prime}=-1 / N_{B}, \beta^{\prime}=1 / N_{B}, \mu_{0 V}=\mu_{0 B}=0, N_{B 1}=N_{B}$, (matched processing) with the weighting coefficients sin

\begin{tabular}{lcc}
\hline \multirow{2}{*}{$y_{B}=y_{V}$} & $\begin{array}{c}\text { with the weighting } \\
\text { coefficients sin }\end{array}$ & $\begin{array}{c}\text { with the weighting } \\
\text { coefficients } \sin ^{2}\end{array}$ \\
\cline { 2 - 3 } & 3.59 & $\xi$ \\
2 & 2.83 & 6.38 \\
3 & 2.24 & 4.97 \\
5 & 1.5 & 3.77 \\
6 & 1.28 & 2,09 \\
7 & 1.13 & 1,6 \\
8 & 1.05 & 1,28 \\
9 & 1.01 & 1.09 \\
& & 1.01
\end{tabular}




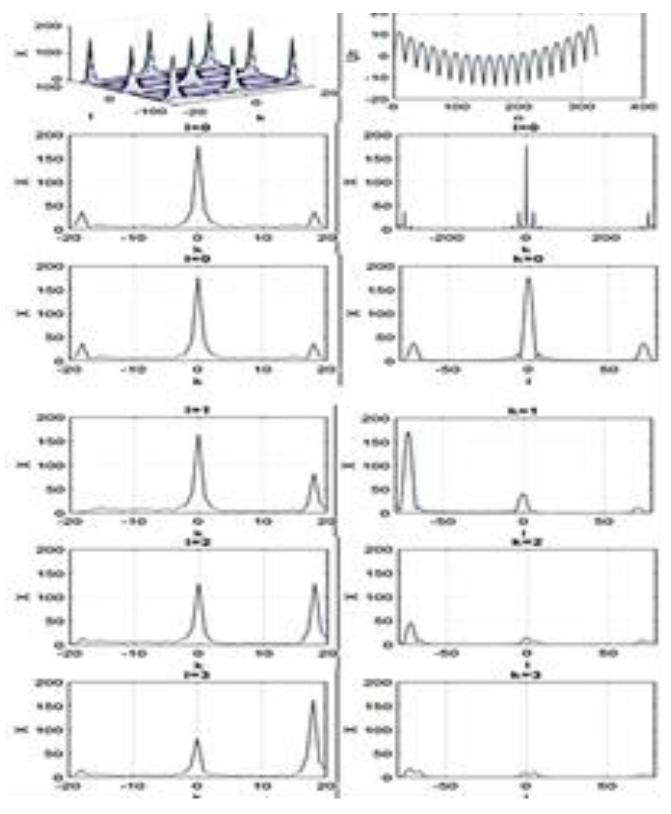

a)

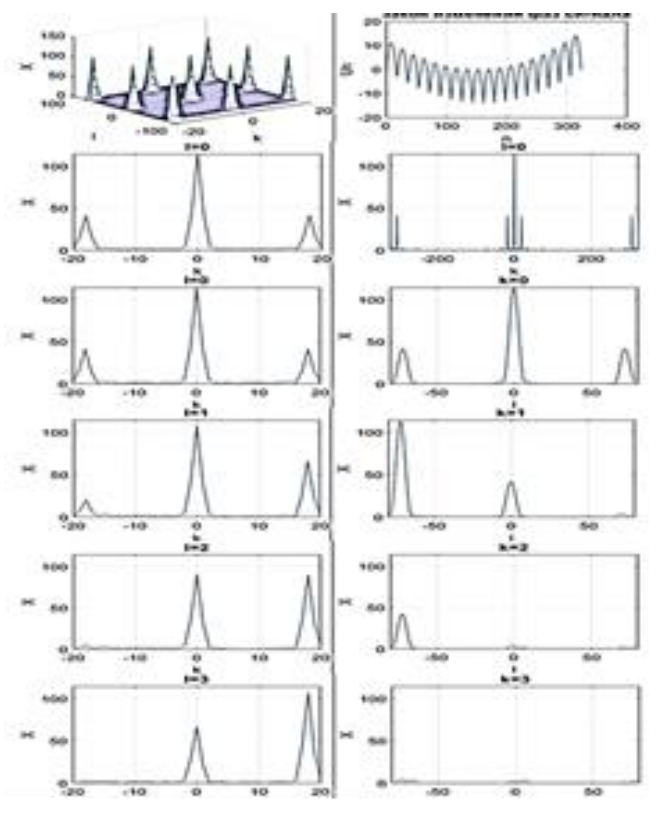

b)

Fig 2. a) The body of the CAF of the periodic multiphase compound signal without an additional AM, $N=324\left(N_{B}=18, N_{V}=18\right) \alpha{ }^{\prime}=-$ $1 / N_{B}, \beta^{\prime}=1 / N_{B}, \mu_{0 \mathrm{~V}}=\mu_{0 B}=0, N_{B 1}=N_{B}$, with the weighting function $\sin \left(y_{B}=y_{V}=2\right)$, phases of the signal, sections of the body of the CAF; b) the body of the AF periodic multiphase compound signal with an additional AM, $N=324\left(N_{B}=18, N_{v}=18\right) \alpha{ }^{\prime}=-1 / N_{B}, \beta^{\prime}=1 / N_{B}, \mu_{0 v}=$ $\mu_{0 B}=0, N_{B 1}=N_{B}$, with the weighting coefficients $\sin \left(y_{B}=y_{V}=2\right)$, phases of the signal, sections of the body of the AF

The calculations were made for three types of the signals, as indicated above:

$$
\begin{aligned}
& \alpha^{\prime}=-1 / \mathrm{N}_{\mathrm{B}}, \beta^{\prime}=1 / \mathrm{N}_{\mathrm{B}}^{2}, \mathrm{~N}=324\left(\mathrm{~N}_{\mathrm{B}}=18, \mathrm{~N}_{\mathrm{V}}=18\right) . \\
& \alpha^{\prime}=-1 / \mathrm{N}_{\mathrm{B}}, \beta^{\prime}=1 / \mathrm{N}_{\mathrm{B}}, \mathrm{N}=324\left(\mathrm{~N}_{\mathrm{B}}=18, \mathrm{~N}_{\mathrm{V}}=18\right), \\
& \alpha^{\prime}=-1 / \mathrm{N}_{\mathrm{B}}, \beta^{\prime}=0, \mathrm{~N}=324\left(\mathrm{~N}_{\mathrm{B}}=18, \mathrm{~N}_{\mathrm{V}}=18\right) .
\end{aligned}
$$

The three kinds of signals with different sets of parameters were compared. The compound multiphase signal with an additional AM N=324 $\left(\mathrm{N}_{\mathrm{B}}=18, \mathrm{~N}_{\mathrm{V}}=18\right), \alpha^{\prime}=-1 / \mathrm{N}_{\mathrm{B}}, \beta^{\prime}=1 / \mathrm{N}_{\mathrm{B}}, \mu_{0 \mathrm{~V}}=\mu_{0 \mathrm{~B}}=0, \mathrm{~N}_{\mathrm{B} 1}=\mathrm{N}_{\mathrm{B}}$, (matched processing) with the weighting function $\sin$ shows the highest ratio of the $C P$ to the side-lobes level. The signal with $\beta^{\prime}=1 / \mathrm{N}_{B}{ }^{2}$ shows almost the same. For example, we demonstrated the cases of signals with parameters $\alpha^{\prime}=-1 / N_{B}, \beta^{\prime}=1 / N_{B}, N=324 \quad\left(N_{B}=18, N_{V}=18\right), y_{V}=y_{B}=1,2$ (Fig.1,2). They have the highest side-lobe level in the FZ region. 


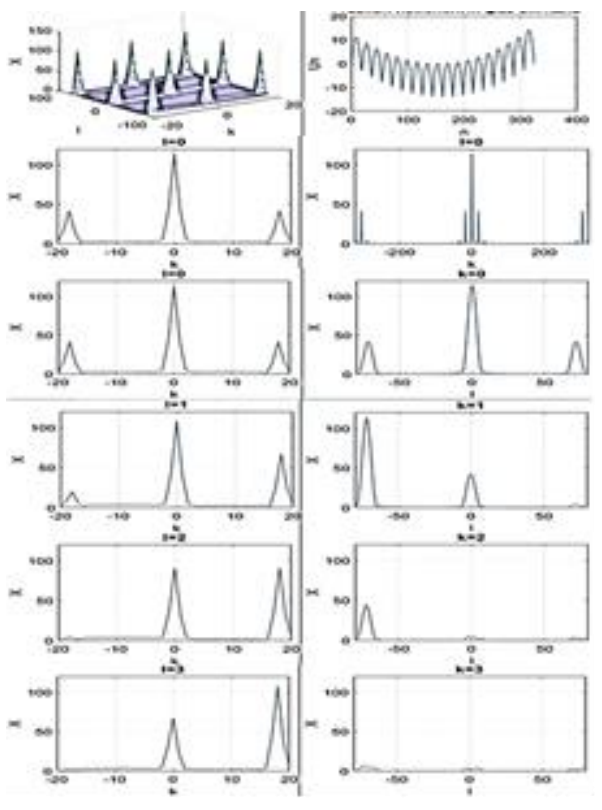

a)

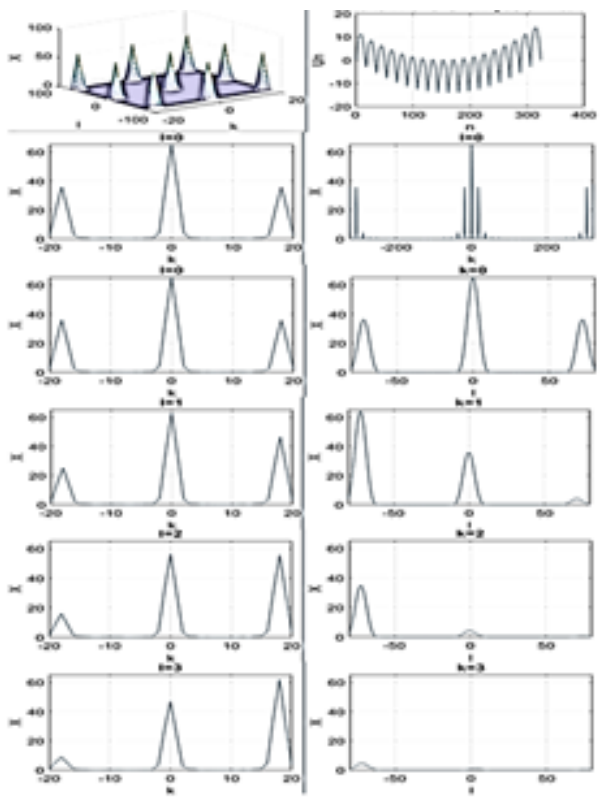

b)

Fig 3. a) The body of the CAF of the periodic multiphase compound signal without an additional $A M, N=324(N B=18, N V=18) \alpha$ $'=-1 / \mathrm{NB}, \beta^{\prime}=1 / \mathrm{NB}, \mu 0 \mathrm{~V}=\mu 0 \mathrm{~B}=0, \mathrm{NB} 1=\mathrm{NB}$, with the weighting function $\sin 2(\mathrm{yB}=\mathrm{yV}=2)$ for $(8)$, phases of the signal, sections of the body of the CAF; b) the body of the AF periodic multiphase compound signal with an additional $A M, N=324(N B=18, N V=18) \alpha{ }^{\prime}=-$ $1 / \mathrm{NB}, \beta^{\prime}=1 / \mathrm{NB}, \mu 0 \mathrm{~V}=\mu 0 \mathrm{~B}=0, \mathrm{NB} 1=\mathrm{NB}$, with the weighting coefficients $\sin 2(\mathrm{yB}=\mathrm{yV}=2)$ for $(8)$, phases of the signal, sections of the body of the AF

Thus, the lowest side-lobes level was given by signals with peak-factor $\xi=3,59$ and $\xi=2,83$ for weighting function sin, while $y_{V}=y_{B}=1,2$. Peak-factor for signals with lowest side-lobes level were $\xi=6,38$ and $\xi=4,97$ (for weighting function $\sin ^{2}$ ). For comparison AF body for rectangular shape of compound signals is presented on Figure 4.

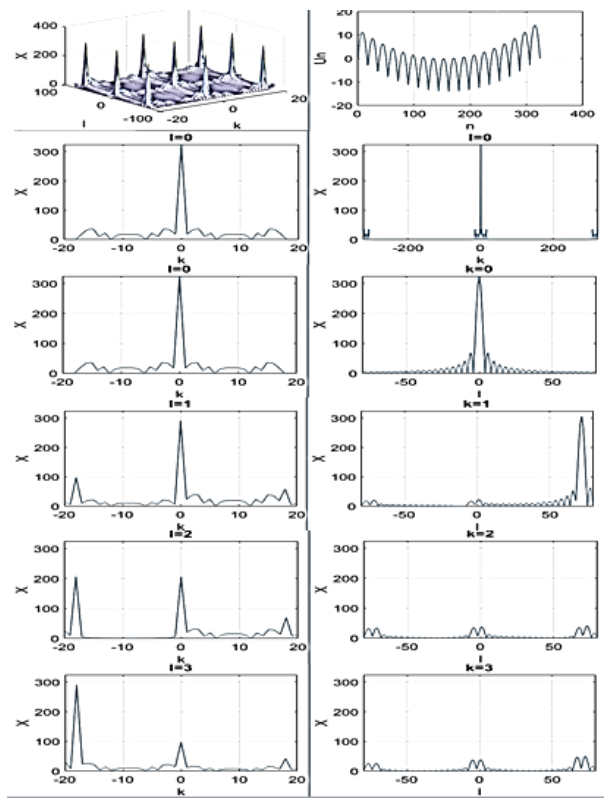

Fig 4. The body of the AF of the periodic multiphase compound signal, $N=324\left(N_{B}=18, N_{V}=18\right), \alpha{ }^{\prime}=-1 / N_{B}, \beta^{\prime}=1 / N_{B}, \mu_{0 V}=\mu_{0 B}=0, N_{B 1}$ $=N_{B}$, phases of the signal, sections of the body of the AF

Undoubtedly, the compound signal on Fig. 4 has the highest side-lobes level in the FZ region. This is a big drawback of this type processing.

The signals with different pulses' quantity of base and external sequence $\left(N_{B}=18, N_{V}=17\right)$ are considered. Parameters of signal are $\alpha^{\prime}=1 / N_{B}, \beta^{\prime}=2 / N_{V}, \mu_{0 V}=1, \mu_{0 B}=0, N_{B 1}=N_{B}$. 


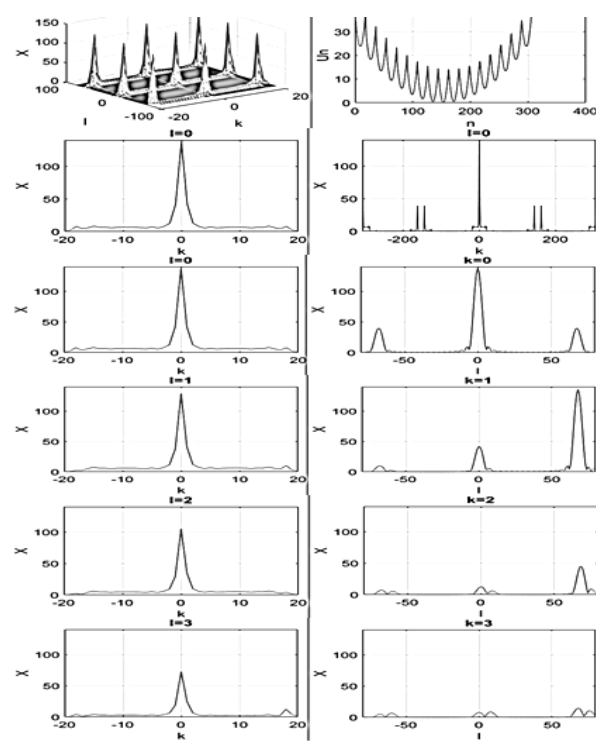

a)

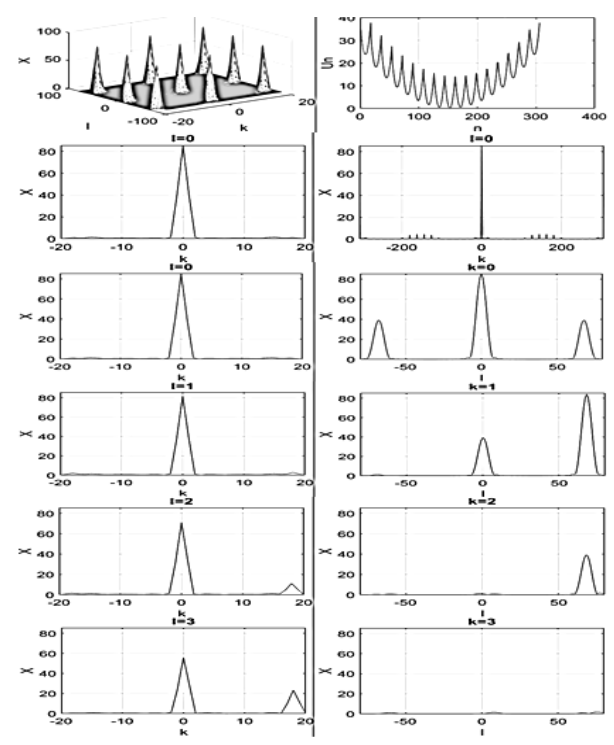

b)

Fig 5. a) The body of the CAF of the periodic multiphase compound signal without an additional AM, $N=306\left(N_{B}=18, N_{V}=17\right)$ $\alpha^{\prime}=1 / N_{B}, \beta^{\prime}=2 / N_{V}, \mu_{0 V}=1, \mu_{0 B}=0, N_{B 1}=N_{B}$, with the weighting function $\sin \left(y_{B}=y_{V}=1\right)$, phases of the signal, sections of the body of the CAF; b) the body of the AF periodic multiphase compound signal with an additional AM, $N=306\left(N_{B}=18, N_{V}=17\right) \alpha^{\prime}=1 / N_{B}, \beta^{\prime}=2 / N_{V}, \mu_{0 V}$ $=1, \mu_{0 B}=0, N_{B 1}=N_{B}$, with the weighting coefficients $\sin \left(y_{B}=y_{V}=1\right)$, phases of the signal, sections of the body of the AF

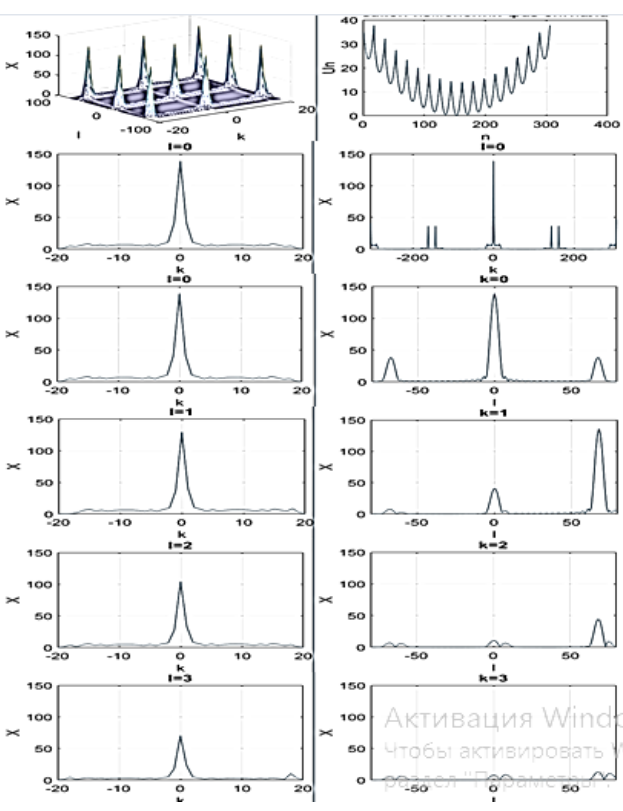

a)

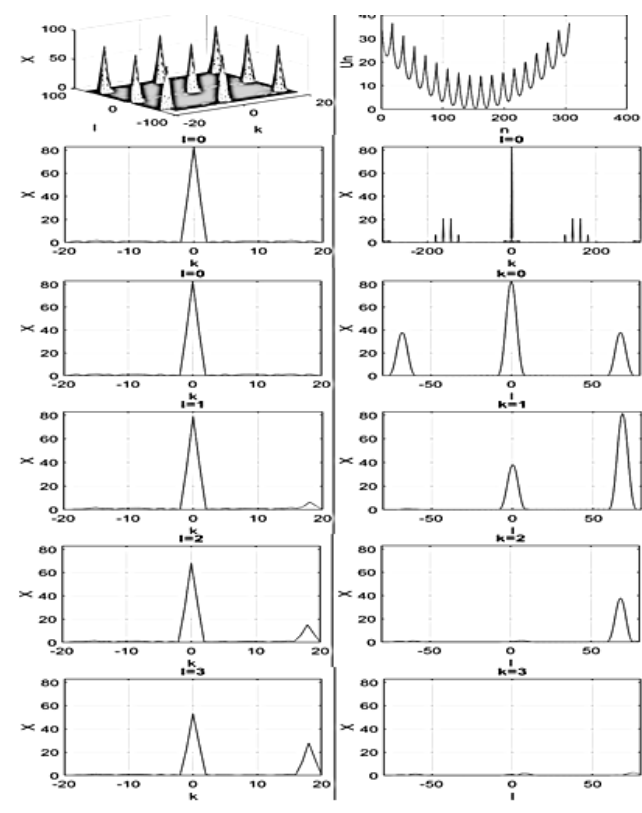

b)

Fig 6. a) The body of the CAF of the periodic multiphase compound signal without an additional $A M, N=306(N B=18, N V=17)$ $\alpha^{\prime}=1 / \mathrm{NB}, \beta^{\prime}=2 / \mathrm{NV}, \mu 0 \mathrm{~V}=1, \mu 0 \mathrm{~B}=0, \mathrm{NB} 1=\mathrm{NB}$, with the weighting function $\sin 2(\mathrm{yB}=\mathrm{yV}=3)$ for $(8)$, phases of the signal, sections of the body of the CAF; b) the body of the AF periodic multiphase compound signal with an additional AM, N=306 (NB=18, NV=17) $\alpha^{\prime}=1 / \mathrm{NB}, \beta^{\prime}=2 / \mathrm{NV}, \mu 0 \mathrm{~V}=1, \mu 0 \mathrm{~B}=0, \mathrm{NB} 1=\mathrm{NB}$, with the weighting coefficients $\sin 2(\mathrm{yB}=\mathrm{yV}=3)$ for $(8)$, phases of the signal, sections of the body of the AF 


\section{Results}

The results of research for these cases are presented in Table 2:

Table 2. The peak-factor dependence from parameters $y_{B}=y_{V}$ for periodic multiphase compound signal with an additional AM, $N=306\left(N_{B}=18, N_{V}=17\right) \alpha^{\prime}=1 / N_{B}, \beta^{\prime}=2 / N_{V}, \mu_{0 v}=1, \mu_{0 B}=0, N_{B 1}=N_{B}$, (matched processing) with the weighting coefficients sin and $\sin ^{2}$

\begin{tabular}{ccc}
\hline \multirow{2}{*}{$y_{\mathrm{B}=y_{\mathrm{V}}}$} & $\begin{array}{c}\text { with the weighting } \\
\text { coefficients sin }\end{array}$ & $\begin{array}{c}\text { with the weighting } \\
\text { coefficients } \sin ^{2}\end{array}$ \\
\cline { 2 - 3 } & \multicolumn{3}{c}{} \\
\hline 1 & 3.58 & 6.36 \\
2 & 2.80 & 4.92 \\
3 & 2.21 & 3.69 \\
5 & 1.46 & 2.01 \\
6 & 1.25 & 1.53 \\
7 & 1.11 & 1.24 \\
8 & 1.03 & 1.07 \\
9 & 1.00 & 1.01 \\
\hline
\end{tabular}

As we can see, if we reduce quantity of pulses in the signal the pick-factor is reduced. The side-lobes level in the FZ region does not hesitate in large limits if we use $N=306$ or $N=324$. But periodic multiphase compound signal with an additional AM with $N=306$ have lower side-lobes level on all sections of the body of the AF (for $l=0$ ), without high side peak.

To summarize, periodic multiphase compound signal with an additional AM have the lowest side-lobes level. A matched processing was used, so we can say this type of signal does not have signal to noise losses.

These results can be used not only for the compound multiphase signals, but also for equivalent compound LFM signals $[6,7,8]$.

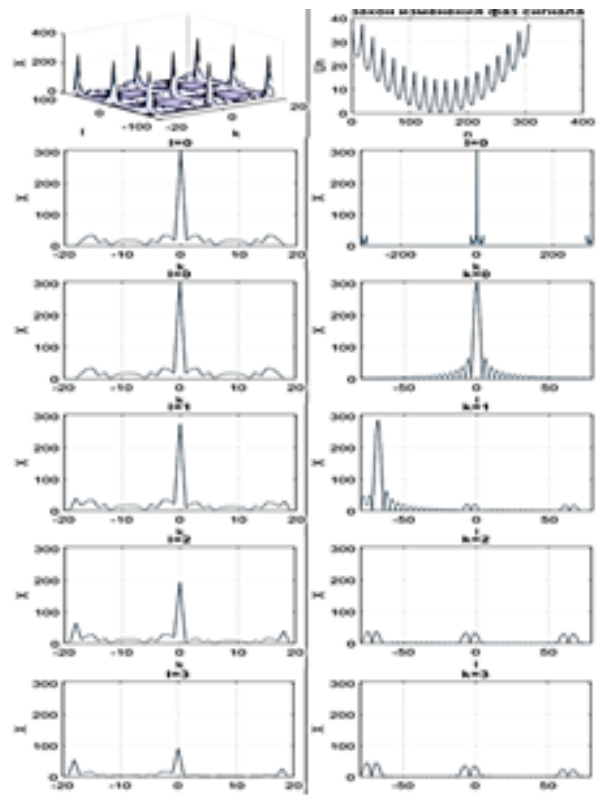

Fig 7. The body of the AF of the periodic multiphase compound signal, $N=306\left(N_{B}=18, N_{V}=17\right) \alpha^{\prime}=1 / N_{B}, \beta^{\prime}=2 / N_{V}, \mu_{0 V}=1, \mu_{0 B}=0, N_{B 1}$ $=N_{B}$, phases of the signal, sections of the body of the $\mathrm{AF}$

To summarize, periodic multiphase compound signals with an additional AM have the lowest side-lobes level in FZ and have good Correlation functions with low sensitivity to the Doppler shifts. A matched processing was used, so this type of signal does not have signal to noise ratio losses.

These results can be used not only for the compound multiphase signals, but also for equivalent compound LFM signals $[6,7,8]$. 


\section{References}

[1] V.M. Koshevyy. Synthesis of Waveform-Filter pairs under Additional Constrains with Group-Complementary Properties IEEE, Radar Conference 2015, May 2015, Arlington,VA (USA), pp 0616-0621.

[2] V.M. Koshevyy, Synthesis compound multiphase signals, Izvestiya VUZ. Radioelectronika (Radioelectronics and Communication Systems), vol. 31, N8, 1988, pp. 56-58.

[3] V. Koshevyy \& O. Pashenko, Improved Compound Multiphase Waveforms with Additional Amplitude Modulation (periodic mode) for Marine Radars, Marin Navigation and Safety of Sea Transportation. Activities in Navigation, 2017 [to be published].

[4] V.M. Koshevyy, V.I. Kuprovskyy. Investigation of properties of compound multiphase signals.Izvestiya VUZ. Radioelectronika (Radioelectronics and Communication Systems), N8, 1991, pp. 63-66.

[5] Ch.E. Cook, M. Bernfeld, Radar Signals. An Introduction to Theory and Application, Artech House, Inc., Boston 1993.

[6] B.L. Lewis, F.F. Kretschmer, Linear frequency modulation derived polyphase pulse compression codes, IEEE Trans. on Aerospace and Electronic Systems Vol. AES-18, N5, Sept. 1982, pp. 637-641.

[7] N. Levanon, E. Mozeson, Radar signals, J. Wiley, NJ, 2004.

[8] V. Koshevyy \& O. Pashenko, Signal Processing Optimization in the FMCW Navigational Radars, Marin Navigation and Safety of Sea Transportation. Activities in Navigation. (edited) Adam Weintrit. CRC Press. 2015 pp. 55-60 